Research Article

Special Issue: Contemporary Spectral Graph Theory

Open Access

Chaohui Chen*, Jiarong Peng, and Tianyuan Chen

\title{
The $A_{\alpha}$-spectral radius of complements of bicyclic and tricyclic graphs with $n$ vertices
}

https://doi.org/10.1515/spma-2021-0147

Received May 29, 2021; accepted August 23, 2021

Abstract: Recently, the extremal problem of the spectral radius in the class of complements of trees, unicyclic graphs, bicyclic graphs and tricyclic graphs had been studied widely. In this paper, we extend the largest ordering of $A_{\alpha}$-spectral radius among all complements of bicyclic and tricyclic graphs with $n$ vertices, respectively.

Keywords: Adjacency spectrum; $A_{\alpha}$-matrix; $A_{\alpha}$-spectral radius; extremal graph

MSC: 05C50; 15A18

\section{Introduction}

Throughout this paper, we only concern with simple undirected graphs. Let $G=(V, E)$ be a graph with vertex set $V(G)=\left\{v_{1}, v_{2}, \ldots, v_{n}\right\}$, and let $d_{G}\left(v_{i}\right)$ be the degree of vertex $v_{i}$ of $G$. We use $\bar{G}$ and $\Delta(G)$ to denote the complement and maximum degree of graph $G$, respectively. The adjacency matrix and diagonal matrix of graph $G$ is denoted by $A(G)$ and $D(G)$, respectively. The $A_{\alpha}$-matrix $A_{\alpha}(G)$ of graph $G$ is denoted by $A_{\alpha}(G)=$ $\alpha D(G)+(1-\alpha) A(G)$, where $\alpha \in[0,1]$. The maximum eigenvalue of $A_{\alpha}(G)$ is called the $A_{\alpha}$-spectral radius of $G$, and denoted by $\rho_{\alpha}(G)$. In particular, $\rho(G)=\rho_{0}(G)$ is equal to the spectral radius (maximum eigenvalue) of $A(G)$. If $G$ has the maximum $A_{\alpha}$-spectral radius among all graphs of a given graph category $\mathcal{G}$, then we call $G$ as a $\rho_{\alpha}$-maximal graph of $\mathcal{G}$.

As usual, let $P_{n}$ and $K_{1, n-1}$ be the path and star with $n$ vertices, respectively. If $G$ has no $2 P_{2}, P_{4}$ or $C_{4}$ (cycle with four vertices) as an induced subgraph, then $G$ is called a split graph (see [6]). Let $T_{1}, T_{2}, U_{1}, U_{2}$, $B_{1}, B_{2}, B_{3}, B_{4}, S_{1}, S_{2}, \ldots, S_{7}$ be the 15 graphs with $n$ vertices as shown in Figure 1.1, and let $\overline{\mathbb{G}}(n ; c)$ be the class of graphs, each of which is the complement of a $c$-cyclic graphs with $n$ vertices, that is,

$$
\overline{\mathbb{G}}(n ; c)=\{\bar{G}: G \text { is a } c \text {-cyclic graph with } n \text { vertices }\} \text {. }
$$

Denoted by $\overline{\mathbb{G}}(n, k ; c)$ the class of graphs, each of which is the complement of a $c$-cyclic graphs with $n$ vertices and maximum degree $k$, that is,

$$
\bar{G}(n, k ; c)=\{\bar{G}: G \text { is a } c \text {-cyclic graph with } n \text { vertices and maximum degree } k\} .
$$

Recently, the extremal problem of the spectral radius in the class of complements of trees, unicyclic graphs, bicyclic graphs and tricyclic graphs had been studied in $[2,7,8]$. It had been shown that

Theorem 1.1. Let $\bar{G}$ be a $\rho$-maximal graph of $\overline{\mathbb{G}}(n ; c)$.

^Corresponding Author: Chaohui Chen: Department of Mathematics, South China Agricultural University, Guangzhou, 510642, China, E-mail: cch956833210@163.com

Jiarong Peng, Tianyuan Chen: Department of Mathematics, South China Agricultural University, Guangzhou, 510642, China The three authors contribute equally to this paper. 
(1) [2] If $c=1$, then $G=U_{1}$;

(2) [7] If $c=2$ and $n \geq 5$, then $G=B_{1}$;

(3) [8] If $c=3$ and $n \geq 8$, then $G=S_{1}$.

Later, the unique $\rho_{\alpha}$-maximal graph among the class of complements of trees, unicyclic graphs and bicyclic graphs was determined by Zhang and Guo [9], that is,

Theorem 1.2. [9] Let $0 \leq \alpha<1$ and $\bar{G}$ be a $\rho_{\alpha}$-maximal graph of $\overline{\mathbb{G}}(n ; c)$.

(1) If $c=0$, then $G=K_{1, n-1}$;

(2) If $c=1$, then $G=U_{1}$;

(3) If $c=2$ and $n \geq 5$, then $G=B_{1}$.

From Theorems 1.1 and 1.2, we can easily see that each of those extremal maximal graphs is the complement of a graph with maximum degree $n-1$. In [3], the present authors and their cooperators extended this fact to the general cases.

Theorem 1.3. [3] Let $0 \leq \alpha<1, \overline{G_{1}}$ and $\overline{G_{2}}$ be a $\rho_{\alpha}$-maximal graph of $\overline{\mathbb{G}}(n, s ; c)$ and $\overline{\mathbb{G}}(n, t ; c)$, respectively. If $0 \leq c \leq n-4$ and $2 \leq s<t \leq n-1$, then $\rho_{\alpha}\left(\overline{G_{2}}\right)>\rho_{\alpha}\left(\overline{G_{1}}\right)$.

Based on Theorem 1.3, the present authors and their cooperators improved Theorem 1.2 (1) and (2) to the following Theorem 1.4, and they also determined the $\rho_{\alpha}$-maximal graphs in the class of complements of tricyclic graphs, that is, Theorem 1.5.

Theorem 1.4. [3] Let $0 \leq \alpha<1$ and $n \geq 4$.

(1) If $\bar{G} \in \overline{\mathbb{G}}(n ; 0) \backslash\left\{\overline{T_{1}}, \overline{T_{2}}\right\}$, then $\rho_{\alpha}(\bar{G})<\rho_{\alpha}\left(\overline{T_{2}}\right)<\rho_{\alpha}\left(\overline{T_{1}}\right)$;

(2) If $\bar{G} \in \overline{\mathbb{G}}(n ; 1) \backslash\left\{\overline{U_{1}}, \overline{U_{2}}\right\}$, then $\rho_{\alpha}(\bar{G})<\rho_{\alpha}\left(\overline{U_{2}}\right)<\rho_{\alpha}\left(\overline{U_{1}}\right)$.

Theorem 1.5. [3] Let $0 \leq \alpha<1, n \geq 7$ and $\bar{G}$ be a $\rho_{\alpha}$-maximal graph of $\overline{\mathbb{G}}(n ; 3)$.

(1) If $0 \leq \alpha<0.5$, then $G=S_{1}$;

(2) If $\alpha=0.5$, then $G \in\left\{S_{1}, S_{2}\right\}$ and $\rho_{0.5}\left(\overline{S_{1}}\right)=\rho_{0.5}\left(\overline{S_{2}}\right)$;

(3) If $0.5<\alpha<1$, then $G=S_{2}$.

By an observation to the above results, it is an interesting problem that: Is it difficult or not to extend those corresponding ordering? Actually, as shown in the later, different from the ordering of largest spectral radius of trees, unicyclic graphs, bicyclic graphs and tricyclic graphs [5], we find the solution to this problem is rather difficult. The main results of this paper are as follows:

Theorem 1.6. Let $0 \leq \alpha \leq 0.87$ and $n \geq 23$. If $\bar{G} \in \overline{\mathbb{G}}(n ; 2) \backslash\left\{\overline{B_{1}}, \overline{B_{2}}, \overline{B_{3}}\right\}$, then

$$
\rho_{\alpha}(\bar{G})<\rho_{\alpha}\left(\overline{B_{3}}\right)<\rho_{\alpha}\left(\overline{B_{2}}\right)<\rho_{\alpha}\left(\overline{B_{1}}\right) .
$$

Theorem 1.7. Let $\bar{G} \in \overline{\mathbb{G}}(n ; 3) \backslash\left\{\overline{S_{1}}, \overline{S_{2}}, \ldots, \overline{S_{6}}\right\}$. If $n \geq 35$ and $0 \leq \alpha \leq 0.8$, then

$$
\rho_{\alpha}(\bar{G})<\rho_{\alpha}\left(\overline{S_{6}}\right)<\rho_{\alpha}\left(\overline{S_{5}}\right)<\rho_{\alpha}\left(\overline{S_{4}}\right)<\rho_{\alpha}\left(\overline{S_{3}}\right)<\min \left\{\rho_{\alpha}\left(\overline{S_{2}}\right), \rho_{\alpha}\left(\overline{S_{1}}\right)\right\} .
$$

The condition $\alpha \leq 0.87$ of Theorem 1.6 is necessary, as we have $\rho_{\alpha}\left(\overline{B_{3}}\right)>20.86307>20.86298>\rho_{\alpha}\left(\overline{B_{2}}\right)$ when $n=23$ and $\alpha=0.88$. Furthermore, the condition $0 \leq \alpha \leq 0.8$ of Theorem 1.7 is also necessary, as we have $\rho_{\alpha}\left(\overline{S_{6}}\right)>32.8447>32.844>\rho_{\alpha}\left(\overline{S_{5}}\right)$ if $n=35$ and $\alpha=0.81$.

\section{The proof of Theorem 1.6}

The following lemma will play an important role in the proof of Theorem 1.6. 

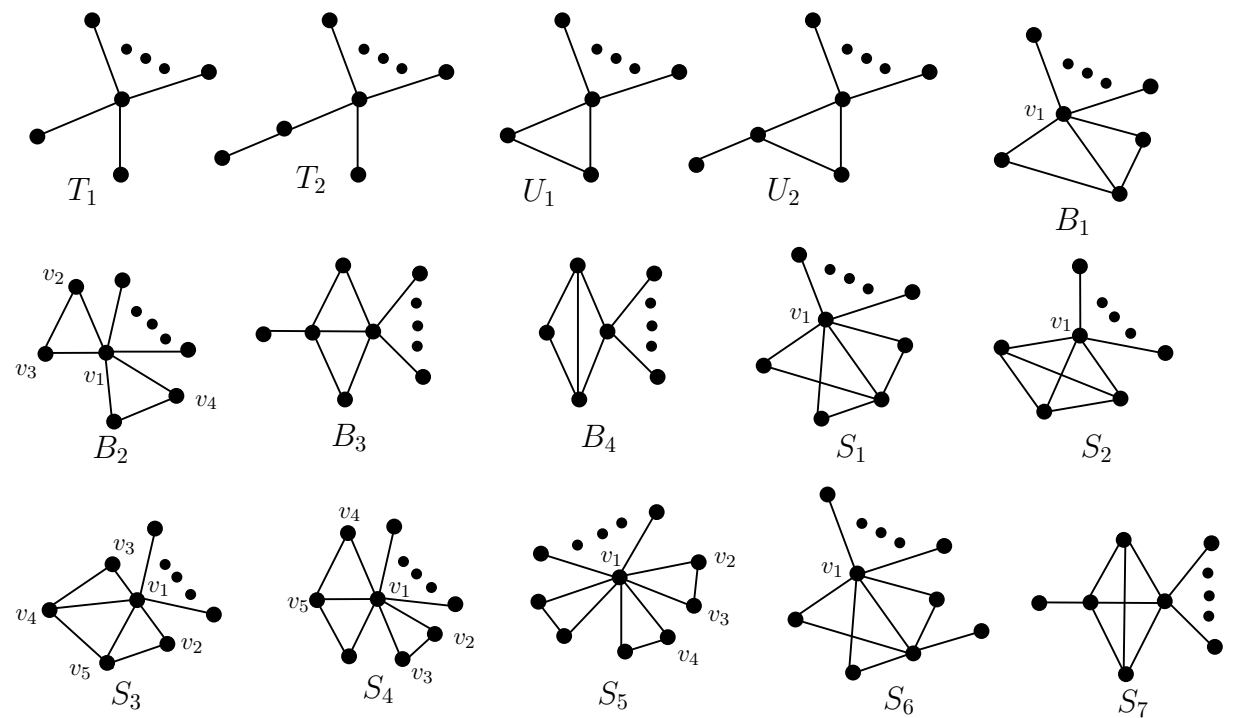

Figure 1.1: The graphs $T_{1}, T_{2}, U_{1}, U_{2}, B_{1}, B_{2}, B_{3}, B_{4}, S_{1}, S_{2}, \ldots, S_{7}$ with $n$ vertices.

Lemma 2.1. [3] Let $\bar{G}$ be a $\rho_{\alpha}$-maximal graph of $\overline{\mathbb{G}}(n, n-2 ; c)$ with $d_{G}\left(u_{0}\right)=n-2, u_{0} v_{0} \notin E(G)$ and $S_{0}=$ $V(G) \backslash\left\{u_{0}\right\}$. If $0 \leq c \leq n-4$, then $G\left[S_{0}\right]$ has unique component $H_{0}$ with at least two vertices such that $H_{0}$ is a split graph with $c+1$ edges. Furthermore, if $d_{H_{0}}\left(v_{0}\right) \geq 2$, then $x$ is adjacent to every vertex of $V\left(H_{0}\right)$ for each vertex $x \in N_{H_{0}}\left(v_{0}\right)$.

By an elementary computation with the application of Equitable partitions (see [1]), $\rho_{\alpha}\left(\overline{B_{2}}\right), \rho_{\alpha}\left(\overline{B_{3}}\right)$ and $\rho_{\alpha}\left(\overline{B_{4}}\right)$ are equal to the maximum root of $\Phi_{1}(x), \Phi_{2}(x)$ and $\Phi_{3}(x)$, respectively, where

$$
\begin{aligned}
& \Phi_{1}(x)=x^{2}-(\alpha(n-1)+n-4) x+\left(n^{2}-3 n-2\right) \alpha+8-2 n, \\
& \Phi_{2}(x)=x^{5}-((3 \alpha+1) n-6 \alpha-5) x^{4}+\left(3 \alpha(\alpha+1) n^{2}-\left(11 \alpha^{2}+18 \alpha+3\right) n+(2 \alpha+11)(2 \alpha+1)\right) x^{3} \\
& -\left(\alpha^{2}(\alpha+3) n^{3}-2 \alpha\left(2 \alpha^{2}+10 \alpha+3\right) n^{2}-\left(3 \alpha^{3}-29 \alpha^{2}-41 \alpha+1\right) n+6 \alpha^{3}+24 \alpha^{2}-78 \alpha+7\right) x^{2} \\
& +\left(\alpha^{3} n^{4}+\alpha^{2}\left(\alpha^{2}-6 \alpha-3\right) n^{3}-\alpha\left(7 \alpha^{3}+5 \alpha^{2}-29 \alpha+2\right) n^{2}+\left(10 \alpha^{4}+68 \alpha^{3}-73 \alpha^{2}+\alpha+4\right) n\right. \\
& \left.-(\alpha+1)\left(58 \alpha^{2}-61 \alpha+19\right)\right) x-(n-5)\left((n-2) \alpha^{2}+\alpha-1\right)\left(\left(n^{2}-3 n-2\right) \alpha^{2}-(2 n-9) \alpha-1\right), \\
& \Phi_{3}(x)=x^{4}-((2 \alpha+1) n-3 \alpha-5) x^{3}+\left(\alpha(\alpha+2) n^{2}-\left(2 \alpha^{2}+10 \alpha+3\right) n-2 \alpha^{2}+8 \alpha+11\right) x^{2} \\
& -\left(\alpha^{2} n^{3}+\alpha\left(\alpha^{2}-4 \alpha-3\right) n^{2}-\left(4 \alpha^{3}+7 \alpha^{2}-20 \alpha+1\right) n+34 \alpha^{2}-37 \alpha+5\right) x \\
& +(n-4)\left(\alpha^{3} n^{2}-\alpha\left(3 \alpha^{2}+\alpha+1\right) n-2 \alpha^{3}+9 \alpha^{2}-3 \alpha+2\right) .
\end{aligned}
$$

Lemma 2.2. If $n \geq 23$ and $0 \leq \alpha \leq 0.87$, then $\rho_{\alpha}\left(\overline{B_{3}}\right)<\rho_{\alpha}\left(\overline{B_{2}}\right)$.

Proof. From the formulas of $\Phi_{1}(x)$ and $\Phi_{2}(x)$, it follows that

$$
\Phi_{2}(x)=\Phi_{1}(x) \Psi_{1}(x)+Y_{1}(x),
$$

where $\Psi_{1}(x)=x^{3}-((2 n-5) \alpha-1) x^{2}+\left(\alpha^{2} n^{2}-\alpha(4 \alpha+1) n-\alpha^{2}+5 \alpha-1\right) x-\alpha^{3} n^{2}+\left(6 \alpha^{3}+3 \alpha^{2}-4 \alpha+2\right) n-5 \alpha^{3}-$ $15 \alpha^{2}+21 \alpha-11$ and $Y_{1}(x)=(1-\alpha) \Psi_{2}(x)$ and $\Psi_{2}(x)=\left((\alpha-1)(\alpha-2) n^{2}+\left(\alpha^{3}-9 \alpha^{2}+23 \alpha-17\right) n-5 \alpha^{3}+20 \alpha^{2}-\right.$ $40 \alpha+33) x-\alpha(\alpha-1)(\alpha-2) n^{3}+\left(8 \alpha^{3}-21 \alpha^{2}+11 \alpha+4\right) n^{2}-\left(13 \alpha^{3}-14 \alpha^{2}-26 \alpha+39\right) n-10 \alpha^{3}+80 \alpha^{2}-147 \alpha+93$.

By (2.1), to complete the proof of this lemma, it suffices to show the following two claims: 
Claim 1. If $x \geq \rho_{\alpha}\left(\overline{B_{2}}\right)$, then $Y_{1}(x)>0$.

Proof. Define $\varphi_{1}(n)=(\alpha-1)(\alpha-2) n^{2}+\left(\alpha^{3}-9 \alpha^{2}+23 \alpha-17\right) n-5 \alpha^{3}+20 \alpha^{2}-40 \alpha+33$. Since $\varphi_{1}^{\prime \prime}(n)=$ $2(\alpha-1)(\alpha-2)>0, \varphi_{1}^{\prime}(n) \geq \varphi_{1}^{\prime}(23)=\alpha^{3}+37 \alpha^{2}-115 \alpha+75=\varphi_{2}(\alpha)$. By Matlab, the two smallest roots of $\varphi_{2}(\alpha)$ are about -39.9273 and 0.95 , we can conclude that $\varphi_{1}^{\prime}(n) \geq \varphi_{2}(\alpha)>0$, as $0 \leq \alpha \leq 0.87$. Thus, $\varphi_{1}(n) \geq \varphi_{1}(23)=2\left(9 \alpha^{3}+171 \alpha^{2}-549 \alpha+350\right)=\varphi_{3}(\alpha)>0$, as the two smallest roots of $\varphi_{3}(\alpha)$ are about -21.8705 and 0.9044 .

Since $\Phi_{1}(n-3)=(n-5)(\alpha-1)<0$, we have $\rho_{\alpha}\left(\overline{B_{2}}\right)>n-3$. When $x \geq n-3$, since $\varphi_{1}(n)>0$, we have

$$
\Psi_{2}(x) \geq \Psi_{2}(n-3)=\varphi_{4}(n),
$$

where $\varphi_{4}(n)=(2-\alpha)(1-\alpha)^{2} n^{3}-(1-\alpha)\left(9 \alpha^{2}-24 \alpha+19\right) n^{2}-\left(21 \alpha^{3}-61 \alpha^{2}+83 \alpha-45\right) n+5 \alpha^{3}+20 \alpha^{2}-27 \alpha-6$.

Since $\varphi_{4}^{\prime \prime \prime}(n)=(2-\alpha)(1-\alpha)^{2}>0$, we have $\varphi_{4}^{\prime \prime}(n) \geq \varphi_{2}^{\prime \prime}(23)=2(1-\alpha)\left(60 \alpha^{2}-183 \alpha+119\right)>0$, and thus $\varphi_{4}^{\prime}(n) \geq \varphi_{4}^{\prime}(23)=-1194 \alpha^{3}+4891 \alpha^{2}-6040 \alpha+2345=\varphi_{5}(\alpha)>0$, as the smallest root of $\varphi_{5}(\alpha)$ is about 0.9021 and $0 \leq \alpha \leq 0.87$. Thus, $\Psi_{2}(x) \geq \varphi_{4}(n) \geq \varphi_{4}(23)=2\left(-3942 \alpha^{3}+16317 \alpha^{2}-20012 \alpha+7656\right)=\varphi_{6}(\alpha)>0$, as the smallest root of $\varphi_{6}(\alpha)$ is about 0.8701 . Combining this with $Y_{1}(x)=(1-\alpha) \Psi_{2}(x)$, we have $Y_{1}(x)>0$, and thus Claim 1 holds.

Claim 2. If $x \geq \rho_{\alpha}\left(\overline{B_{2}}\right)$, then $\Psi_{1}(x)>0$.

Proof. When $x>n-3$, since $\Psi_{1}^{\prime \prime}(x)=2(3 x+5 \alpha-2 n \alpha+1) \geq 2((3-2 \alpha) n+5 \alpha-8) \geq 2(23(3-2 \alpha)+5 \alpha-8)=2(61-$ $41 \alpha)>0$, we have $\Psi_{1}^{\prime}(x) \geq \Psi_{1}^{\prime}(n-3)=\varphi_{7}(n)$, where $\varphi_{7}(n)=(\alpha-1)(\alpha-3) n^{2}-\left(4 \alpha^{2}-21 \alpha+16\right) n-\alpha^{2}-25 \alpha+20$. Note that $\varphi_{7}^{\prime \prime}(n)=2(\alpha-1)(\alpha-3)>0$. Thus, $\varphi_{7}^{\prime}(n) \geq \varphi_{7}^{\prime}(23)=42 \alpha^{2}-163 \alpha+122>0$, which implies that $\varphi_{7}(n) \geq \varphi_{7}(23)=436 \alpha^{2}-1658 \alpha+1239>0$. Therefore,

$$
\Psi_{1}(x) \geq \Psi_{1}(n-3)=\varphi_{8}(n)
$$

where $\varphi_{8}(n)=(1-\alpha)^{2} n^{3}+(1-\alpha)\left(\alpha^{2}+8 \alpha-8\right) n^{2}+\left(6 \alpha^{3}+14 \alpha^{2}-44 \alpha+22\right) n-5 \alpha^{3}-12 \alpha^{2}+51 \alpha-26$.

Since $\varphi_{8}^{\prime \prime \prime}(n)=6(1-\alpha)^{2}>0$, we have $\varphi_{8}^{\prime \prime}(n) \geq \varphi_{8}^{\prime \prime}(23)=2(1-\alpha)\left(\alpha^{2}-61 \alpha+61\right)>0$, and thus $\varphi_{8}^{\prime}(n) \geq$ $\varphi_{8}^{\prime}(23)=-40 \alpha^{3}+1279 \alpha^{2}-2482 \alpha+1241=\varphi_{9}(\alpha)>0$, as the smallest root of $\varphi_{9}(\alpha)$ is about 0.9733 . This implies that $\Psi_{1}(x) \geq \varphi_{8}(n) \geq \varphi_{8}(23)=-396 \alpha^{3}+8774 \alpha^{2}-16831 \alpha+8415=\varphi_{10}(\alpha)>0$, as the smallest root of $\varphi_{10}(\alpha)$ is about 0.9538 . Now, we can conclude that Claim 2 holds.

By $\Phi_{2}(x)$ and $\Phi_{3}(x)$, we can deduce the following Lemma 2.3.

Lemma 2.3. If $n \geq 23$ and $0 \leq \alpha \leq 0.87$, then $\rho_{\alpha}\left(\overline{B_{4}}\right)<\rho_{\alpha}\left(\overline{B_{3}}\right)$.

Proof. By the formulas of $\Phi_{2}(x)$ and $\Phi_{3}(x)$, we have

$$
\Phi_{2}(x)+Y_{2}(x)=(x-n+2) \Phi_{3}(x) \text {, and } Y_{2}(x)=((\alpha-1)(n-3)-1) \Phi_{3}(x)+Y_{3}(x),
$$

where $Y_{2}(x)=((\alpha-1)(n-3)-1) x^{4}-\left(\left(2 \alpha^{2}-\alpha-1\right) n^{2}-\left(9 \alpha^{2}+\alpha-7\right) n+6 \alpha^{2}+10 \alpha-10\right) x^{3}+\left(\alpha(\alpha+2)(\alpha-1) n^{3}-\left(5 \alpha^{3}+\right.\right.$ $\left.\left.12 \alpha^{2}-11 \alpha-3\right) n^{2}+\left(\alpha^{3}+34 \alpha^{2}-7 \alpha-17\right) n+6 \alpha^{3}-14 \alpha^{2}-25 \alpha+24\right) x^{2}-\left(\alpha^{2}(\alpha-1) n^{4}+\alpha\left(\alpha^{3}-8 \alpha^{2}+3 \alpha+3\right) n^{3}-\left(7 \alpha^{4}-\right.\right.$ $\left.\left.8 \alpha^{3}-29 \alpha^{2}+27 \alpha-1\right) n^{2}+\left(10 \alpha^{4}+50 \alpha^{3}-134 \alpha^{2}+77 \alpha-5\right) n-66 \alpha^{3}+107 \alpha^{2}-44 \alpha-1\right) x+\alpha^{3}(\alpha-1) n^{4}-\alpha\left(10 \alpha^{3}-\right.$ $\left.8 \alpha^{2}-1\right) n^{3}+\left(29 \alpha^{4}-9 \alpha^{3}-10 \alpha^{2}-\alpha-2\right) n^{2}-\left(16 \alpha^{4}+58 \alpha^{3}-75 \alpha^{2}+30 \alpha-13\right) n-20 \alpha^{4}+116 \alpha^{3}-137 \alpha^{2}+74 \alpha-21$, and $Y_{3}(x)=\alpha(3 \alpha-1) x^{3}-\left(\alpha(\alpha+1)(3 \alpha-1) n-14 \alpha^{2}+8 \alpha-2\right) x^{2}+\left(\alpha^{2}(3 \alpha-1) n^{2}+\left(2 \alpha^{4}-3 \alpha^{3}-11 \alpha^{2}+8 \alpha-\right.\right.$ 2) $\left.n-36 \alpha^{3}+72 \alpha^{2}-45 \alpha+11\right) x-(2 \alpha-1)\left(\alpha^{2}(\alpha+1) n^{2}-\left(3 \alpha^{3}+4 \alpha^{2}+4 \alpha-1\right) n-2 \alpha^{3}+3 \alpha^{2}+16 \alpha-5\right)$.

Let $\beta_{1}$ be the maximum root of $Y_{2}(x)$.

Claim 3. $Y_{2}(n-2.26)>0$.

Proof. Let $6250000 Y_{2}(n-2.26)=1625000(1-\alpha)^{3} n^{4}-2500(1-\alpha)^{2}\left(1850 \alpha^{2}-22869 \alpha+18757\right) n^{3}-50(1-$ $\alpha)\left(397500 \alpha^{3}-7724750 \alpha^{2}+12555294 \alpha-5285491\right) n^{2}+\left(41250000 \alpha^{4}+618672500 \alpha^{3}-1835793650 \alpha^{2}+\right.$ 
$1656883211 \alpha-481324711) n-125000000 \alpha^{4}-15715000 \alpha^{3}+641079100 \alpha^{2}-724756083 \alpha+225411222=$ $\varphi_{1}(n)$. Since $\varphi_{1}^{(4)}(n)=39000000(1-\alpha)^{3}>0$, we have $\varphi_{1}^{\prime \prime \prime}(n) \geq \varphi_{1}^{\prime \prime \prime}(23)=-15000(1-\alpha)^{2}\left(1850 \alpha^{2}+36931 \alpha-\right.$ 41043) $>0$, and thus $\varphi_{1}^{\prime \prime}(n) \geq \varphi_{1}^{\prime \prime}(23)=100(1-\alpha)\left(5985000 \alpha^{3}+25599200 \alpha^{2}-75255594 \alpha+43728841\right)=$ $100(1-\alpha) \varphi_{2}(\alpha)$. Since the two smallest roots of $\varphi_{2}(\alpha)$ are -6.4149 and 1.0102 , we have $\varphi_{2}(\alpha)>0$, as $0 \leq \alpha \leq 0.87$. This implies that $\varphi_{1}^{\prime \prime}(n)>0$. Now, we have $\varphi_{1}^{\prime}(n) \geq \varphi_{1}^{\prime}(23)=-6384375000 \alpha^{4}+$ $8264505000 \alpha^{3}+18841020050 \alpha^{2}-37063869789 \alpha+16342407089=\varphi_{3}(\alpha)>0$, as the two smallest roots of $\varphi_{3}(\alpha)$ are -1.9871 and 0.9977. Thus, 6250000 $Y_{2}(n-2.26)=\varphi_{1}(n) \geq \varphi_{1}(23)=-44934750000 \alpha^{4}+$ $152801172500 \alpha^{3}-158999173550 \alpha^{2}+37969822020 \alpha+13156757319=\varphi_{4}(\alpha)>0$, as the two smallest roots of $\varphi_{4}(\alpha)$ are -0.1821 and 0.9959 .

Claim 4. If $x \geq n-2$, then $Y_{2}(x)<0$.

Proof. Since $Y_{2}^{(4)}(x)=24((\alpha-1)(n-3)-1)<0$, we have $Y_{2}^{\prime \prime \prime}(x) \leq Y_{2}^{\prime \prime \prime}(n-2)=-6(1-\alpha)(3-2 \alpha) n^{2}+\left(54 \alpha^{2}-\right.$ $114 \alpha+54) n-36 \alpha^{2}+84 \alpha-36=\varphi_{5}(n)$. Note that $\varphi_{5}^{\prime \prime}(n)=-12(1-\alpha)(3-2 \alpha)<0$. Thus, $\varphi_{5}^{\prime}(n) \leq \varphi_{5}^{\prime}(23)=$ $-498 \alpha^{2}+1266 \alpha-774<0$, and so $Y_{2}^{\prime \prime \prime}(x) \leq \varphi_{5}(n) \leq \varphi_{5}(23)=-5142 \alpha^{2}+13332 \alpha-8316<0$.

This follows that $Y_{2}^{\prime \prime}(x) \leq Y_{2}^{\prime \prime}(n-2)=-2(3-\alpha)(1-\alpha)^{2} n^{3}+2(1-\alpha)\left(5 \alpha^{2}-22 \alpha+12\right) n^{2}+\left(2 \alpha^{3}-76 \alpha^{2}+106 \alpha-\right.$ 34) $n+12 \alpha^{3}+44 \alpha^{2}-74 \alpha+24=\varphi_{6}(n)$. Since $\varphi_{6}^{\prime \prime \prime}(n)=-12(3-\alpha)(1-\alpha)^{2}<0$, we have $\varphi_{6}^{\prime \prime}(n) \leq \varphi_{6}^{\prime \prime}(23)=$ $4(\alpha-1)\left(64 \alpha^{2}-254 \alpha+195\right)<0$, and thus $\varphi_{6}^{\prime}(n) \leq \varphi_{6}^{\prime}(23)=2716 \alpha^{3}-13462 \alpha^{2}+19196 \alpha-8452=\varphi_{7}(\alpha)<0$, as the smallest root of $\varphi_{7}(\alpha)$ is about 1.0051 . Thus, $Y_{2}^{\prime \prime}(x) \leq \varphi_{6}(n) \leq \varphi_{6}(23)=19102 \alpha^{3}-94808 \alpha^{2}+136730 \alpha-$ $61064=\varphi_{8}(\alpha)<0$, as the smallest root of $\varphi_{8}(\alpha)$ is about 1.0099 .

Thus, $Y_{2}^{\prime}(x) \leq Y_{2}^{\prime}(n-2)=(1-\alpha) \varphi_{9}(n)$, where $\varphi_{9}(n)=-(1-\alpha)^{2} n^{4}-(1-\alpha)\left(\alpha^{2}+8 \alpha-5\right) n^{3}-\left(7 \alpha^{3}+21 \alpha^{2}-\right.$ $42 \alpha+17) n^{2}+\left(10 \alpha^{3}+52 \alpha^{2}-98 \alpha+45\right) n+3(1-\alpha)(14 \alpha-13)$. Since $\varphi_{9}^{(4)}(n)=-24(1-\alpha)^{2}<0$, we have $\varphi_{9}^{\prime \prime \prime}(n) \leq \varphi_{9}^{\prime \prime \prime}(23)=6(\alpha-1)\left(\alpha^{2}-84 \alpha+87\right)<0$, and thus $\varphi_{9}^{\prime \prime}(n) \leq \varphi_{9}^{\prime \prime}(23)=124 \alpha^{3}-5424 \alpha^{2}+10986 \alpha-$ $5692=\varphi_{10}(\alpha)<0$, as the smallest root of $\varphi_{10}(\alpha)$ is about 1.0136. This implies that $\varphi_{9}^{\prime}(n) \leq \varphi_{9}^{\prime}(23)=$ $1275 \alpha^{3}-38473 \alpha^{2}+78539 \alpha-41470=\varphi_{11}(\alpha)<0$, as the smallest root of $\varphi_{11}(\alpha)$ is about 1.0293. Therefore, $\varphi_{9}(n) \leq \varphi_{9}(23)=8694 \alpha^{3}-204627 \alpha^{2}+421556 \alpha-227003=\varphi_{12}(\alpha)<0$, as the smallest root of $\varphi_{12}(\alpha)$ is about 1.0456 .

Now, we can conclude that $Y_{2}(x) \leq Y_{2}(n-2)=(1-\alpha)^{2} \varphi_{13}(n)$, where $\varphi_{13}(n)=-(1-\alpha)(6-\alpha) n^{3}+$ $\left(5 \alpha^{2}-51 \alpha+37\right) n^{2}+\left(4 \alpha^{2}+96 \alpha-64\right) n-20 \alpha^{2}-32 \alpha+25$. Since $\varphi_{13}^{\prime \prime \prime}(n)=-6(1-\alpha)(6-\alpha)<0$, we have $\varphi_{13}^{\prime \prime}(n) \leq \varphi_{13}^{\prime \prime}(23)=-128 \alpha^{2}+864 \alpha-754<0$, and thus $\varphi_{13}^{\prime}(n) \leq \varphi_{13}^{\prime}(23)=-1353 \alpha^{2}+8859 \alpha-7884<0$. This follows that $Y_{2}(x) \leq(1-\alpha)^{2} \varphi_{13}(n) \leq(1-\alpha)^{2} \varphi_{13}(23)=(1-\alpha)^{2}\left(-9450 \alpha^{2}+60366 \alpha-54876\right)<0$.

Claim 5. For $n \geq 24$ and $0.81 \leq \alpha \leq 0.87, n-2.16<\beta_{1}<n-2$.

Proof. Combining with Claim 4 and $(\alpha-1)(n-3)-1<0$, we just need to show that $390625 Y_{2}(n-$ $2.16)=62500(1-\alpha)^{3} n^{4}-625(1-\alpha)^{2}\left(525 \alpha^{2}-5191 \alpha+4298\right) n^{3}+\left(1515625 \alpha^{4}-24753125 \alpha^{3}+\right.$ $\left.60460700 \alpha^{2}-52863625 \alpha+15640425\right) n^{2}+4\left(546875 \alpha^{4}+9252500 \alpha^{3}-27051725 \alpha^{2}+24249539 \alpha-\right.$ $6999289) n-7812500 \alpha^{4}+560000 \alpha^{3}+34870225 \alpha^{2}-39924418 \alpha+12333237=\varphi_{14}(n)>0$. Since $\varphi_{14}^{(4)}(n)=1500000(1-\alpha)^{3}>0$, we have $\varphi_{14}^{\prime \prime \prime}(n) \geq \varphi_{14}^{\prime \prime \prime}(24)=-3750(1-\alpha)^{2}\left(525 \alpha^{2}+4409 \alpha-5302\right)>0$, and thus $\varphi_{14}^{\prime \prime}(n) \geq \varphi_{14}^{\prime \prime}(24)=50(1-\alpha)\left(884375 \alpha^{3}-719300 \alpha^{2}-1688728 \alpha+1529217\right)=50(1-\alpha) \varphi_{15}(\alpha)>0$, as the two smallest roots of $\varphi_{15}(\alpha)$ are about -1.4102 and 1.0124. This implies that $\varphi_{14}^{\prime}(n) \geq \varphi_{14}^{\prime}(24)=$ $-492062500 \alpha^{4}+2133140000 \alpha^{3}-3259493300 \alpha^{2}+2081504156 \alpha-463096756=\varphi_{16}(\alpha)>0$, as the two smallest roots of $\varphi_{16}(\alpha)$ are about 0.5078 and 0.9987 . Now, we can conclude that $390625 Y_{2}(n-2.16)=$ $\varphi_{14}(n) \geq \varphi_{14}(24)=-3618312500 \alpha^{4}+19817240000 \alpha^{3}-36899932175 \alpha^{2}+28750263326 \alpha-8049433707>$ 0 . Thus, $n-2.16<\beta_{1}<n-2$.

Claim 6. If $0 \leq \alpha<\frac{113}{139} \approx 0.8129$, then $Y_{3}(n-2.26)>0$.

Proof. It suffices to show that $125000 Y_{3}(n-2.26)=\varphi_{17}(n)>0$, where $\varphi_{17}(n)=2500 \alpha(1-\alpha)(113-$ $139 \alpha) n^{2}+50\left(3700 \alpha^{4}-98857 \alpha^{3}+183333 \alpha^{2}-107838 \alpha+18700\right) n+500000 \alpha^{4}+9170000 \alpha^{3}-19355391 \alpha^{2}+$ $12297797 \alpha-2455600$. Since $\varphi_{17}^{\prime \prime}(n)=5000 \alpha(1-\alpha)(113-139 \alpha)>0$, we have $\varphi_{17}^{\prime}(n) \geq \varphi_{17}^{\prime}(23)=$ 
$50\left(3700 \alpha^{4}+220843 \alpha^{3}-396267 \alpha^{2}+152062 \alpha+18700\right)=\varphi_{18}(\alpha)>0$, as the second and third roots of $\varphi_{18}(\alpha)$ are about -0.0971 and 0.8289 . Thus, $125000 Y_{3}(n-2.26)=\varphi_{17}(n) \geq \varphi_{17}(23)=4755000 \alpha^{4}+$ $79311950 \alpha^{3}-141792441 \alpha^{2}+37726597 \alpha+19049400=\varphi_{19}(\alpha)>0$, as the second and third roots of $\varphi_{19}(\alpha)$ are about -0.2462 and 0.8432 .

Claim 7. $Y_{3}\left(\beta_{1}\right)>0$.

Proof. Let $\varphi_{20}(\alpha)=\alpha(3 \alpha-1)$. From Claims 3 and 4, we have

$$
n-2.26<\beta_{1}<n-2
$$

Case 1. $\alpha=0$. Then $Y_{3}(x)=2 x^{2}-(2 n-11) x+n-5$. When $x \geq n-2.26$, since $Y_{3}^{\prime}(x) \geq Y_{3}^{\prime}(n-2.26)=2 n+1.96>0$, we have $625 Y_{3}(x) \geq 625 Y_{3}(n-2.26)=4675 n-12278>0$. Since $\beta_{1}>n-2.26$ by $(2.3), Y_{3}\left(\beta_{1}\right)>0$.

Case 2. $0<\alpha<\frac{1}{3}$. We first show that $Y_{3}(n-2)=(1-\alpha) \varphi_{21}(n)>0$, where $\varphi_{21}(n)=2 \alpha(1-\alpha) n^{2}-\left(2 \alpha^{3}-\right.$ $\left.35 \alpha^{2}+35 \alpha-8\right) n+(1-2 \alpha)\left(2 \alpha^{2}+35 \alpha-19\right)$. Since $\varphi_{21}^{\prime \prime}(n)=4 \alpha(1-\alpha)>0$, we have $\varphi_{21}^{\prime}(n) \geq \varphi_{21}^{\prime}(23)=$ $-2 \alpha^{3}-57 \alpha^{2}+57 \alpha+8>0$. This follows that $\varphi_{21}(n) \geq \varphi_{21}(23)=-50 \alpha^{3}-321 \alpha^{2}+326 \alpha+165>0$, and thus $Y_{3}(n-2)>0$.

We second show that either $Y_{3}(7)<0$ or $Y_{3}(\alpha n)<0$.

If $0<\alpha \leq 0.3$, then $Y_{3}(7)=-2 \alpha^{2}\left(\alpha^{2}-10 \alpha+3\right) n^{2}+\left(20 \alpha^{4}-163 \alpha^{3}-171 \alpha^{2}+99 \alpha-13\right) n+4 \alpha^{4}-260 \alpha^{3}+$ $2190 \alpha^{2}-1024 \alpha+170=\varphi_{22}(n)$. Since $\varphi_{22}^{\prime \prime}(n)=-4 \alpha^{2}\left(\alpha^{2}-10 \alpha+3\right)<0$, we have $\varphi_{22}^{\prime}(n) \leq \varphi_{22}^{\prime}(23)=$ $-72 \alpha^{4}+757 \alpha^{3}-447 \alpha^{2}+99 \alpha-13<0$, and thus $Y_{3}(7)=\varphi_{22}(n) \leq \varphi_{22}(23)=-594 \alpha^{4}+6571 \alpha^{3}-4917 \alpha^{2}+$ $1253 \alpha-129<0$.

If $0.3<\alpha<\frac{1}{3}$, then $Y_{3}(\alpha n)=-\alpha(1-\alpha)\left(2 \alpha^{3}+11 \alpha^{2}-9 \alpha+2\right) n^{2}-\left(30 \alpha^{4}-77 \alpha^{3}+41 \alpha^{2}-5 \alpha-1\right) n-$ $(1-2 \alpha)\left(2 \alpha^{3}-3 \alpha^{2}-16 \alpha+5\right)=\varphi_{23}(n)$. Since $\varphi_{23}^{\prime \prime}(n)=-2 \alpha(1-\alpha)\left(2 \alpha^{3}+11 \alpha^{2}-9 \alpha+2\right)<0$, we have $\varphi_{23}^{\prime}(n) \leq \varphi_{23}^{\prime}(23)=92 \alpha^{5}+384 \alpha^{4}-843 \alpha^{3}+465 \alpha^{2}-87 \alpha+1<0$, and thus $Y_{3}(\alpha n)=\varphi_{23}(n) \leq \varphi_{23}(23)=$ $1058 \alpha^{5}+4075 \alpha^{4}-8817 \alpha^{3}+4847 \alpha^{2}-917 \alpha+18<0$.

Combining this with $\varphi_{20}(\alpha)=\alpha(3 \alpha-1)<0$ and (2.3), we have $\max \{7, \alpha n\}<n-2.26<\beta_{1}<n-2$, and thus $Y_{3}\left(\beta_{1}\right)>0$, as $Y_{3}(n-2.26)>0$ by Claim 6 .

Case 3. $\alpha=\frac{1}{3}$. Since $\alpha=\frac{1}{3}, 81 Y_{3}(x)=4\left(18 x^{2}-(13 n-54) x+n^{2}-6 n+4\right)=4 \varphi_{24}(x)$. If $x \geq n-2.26$, then $25 \varphi_{24}^{\prime}(x)=25(36 x-13 n+54) \geq 25 \varphi_{24}^{\prime}(n-2.26)=575 n-684>0$, and thus $1250 \varphi_{24}(x) \geq 1250 \varphi_{24}(n-$ 2.26) $=7500 n^{2}-4975 n-32629>0$. Combining this with $\beta_{1}>n-2.26$ by $(2.3)$, we have $Y_{3}\left(\beta_{1}\right)>0$.

Case 4. $\frac{1}{3}<\alpha<\frac{113}{139} \approx 0.8129$. Since $\beta_{1}>n-2.26$ by (2.3), it suffices to show that

$$
\text { If } x \geq n-2.26 \text {, then } Y_{3}(x)>0 \text {. }
$$

Since $Y_{3}^{\prime \prime \prime}(x)=6 \varphi_{20}(\alpha)>0$, we have $25 Y_{3}^{\prime \prime}(x) \geq 25 Y_{3}^{\prime \prime}(n-2.26)=50 \alpha(3 \alpha-1)(2-\alpha) n-317 \alpha^{2}-61 \alpha+100=$ $\varphi_{25}(n) \geq \varphi_{25}(23)=-3450 \alpha^{3}+7733 \alpha^{2}-2361 \alpha+100>0$, and thus $2500 Y_{3}^{\prime}(x) \geq 2500 Y_{3}^{\prime}(n-2.26)=$ $2500 \alpha(3 \alpha-1)(1-\alpha) n^{2}+\left(5000 \alpha^{4}+26400 \alpha^{3}-36600 \alpha^{2}+2600 \alpha+5000\right) n-90000 \alpha^{3}+136721 \alpha^{2}-60407 \alpha+$ $4900=\varphi_{26}(n)$. Note that $\varphi_{26}^{\prime \prime}(n)=5000 \alpha(3 \alpha-1)(1-\alpha)>0$. Thus, $\varphi_{26}^{\prime}(n) \geq \varphi_{26}^{\prime}(23)=200\left(25 \alpha^{4}-1593 \alpha^{3}+\right.$ $\left.2117 \alpha^{2}-562 \alpha+25\right)>0$, which implies that $2500 Y_{3}^{\prime}(x) \geq \varphi_{26}(n) \geq \varphi_{26}(23)=115000 \alpha^{4}-3450300 \alpha^{3}+$ $4584921 \alpha^{2}-1323107 \alpha+119900>0$. Now, we can conclude that $125000 Y_{3}(x) \geq 125000 Y_{3}(n-2.26)>0$ by Claim 6 and thus (2.4) holds.

Case 5. $0.81<\alpha \leq 0.87$ and $n \geq 24$. By Claim 5, it suffices to show that

$$
\text { If } x \geq n-2.16 \text {, then } Y_{3}(x)>0 \text {. }
$$

Since $Y_{3}^{\prime \prime \prime}(x)=6 \varphi_{20}(\alpha)>0$, we have $25 Y_{3}^{\prime \prime}(x) \geq 25 Y_{3}^{\prime \prime}(n-2.16)=50 \alpha(3 \alpha-1)(2-\alpha) n-272 \alpha^{2}-76 \alpha+100=$ $\varphi_{27}(n) \geq \varphi_{27}(24)=-3600 \alpha^{3}+8128 \alpha^{2}-2476 \alpha+100>0$, and thus $625 Y_{3}^{\prime}(x) \geq 625 Y_{3}^{\prime}(n-2.16)=625 \alpha(3 \alpha-$ 1) $(1-\alpha) n^{2}+25\left(50 \alpha^{4}+249 \alpha^{3}-331 \alpha^{2}+16 \alpha+50\right) n-22500 \alpha^{3}+33444 \alpha^{2}-15273 \alpha+1475=\varphi_{28}(n)$. Since $\varphi_{28}^{\prime \prime}(n)=1250 \alpha(3 \alpha-1)(1-\alpha)>0$, we have $\varphi_{28}^{\prime}(n) \geq \varphi_{28}^{\prime}(24)=25\left(50 \alpha^{4}-3351 \alpha^{3}+4469 \alpha^{2}-1184 \alpha+50\right)>0$, and thus $625 Y_{3}^{\prime}(x) \geq \varphi_{28}(n) \geq \varphi_{28}(24)=30000 \alpha^{4}-953100 \alpha^{3}+1274844 \alpha^{2}-365673 \alpha+31475>0$.

This follows that $15625 Y_{3}(x) \geq 15625 Y_{3}(n-2.16)=1250 \alpha(27-31 \alpha)(1-\alpha) n^{2}+25\left(1050 \alpha^{4}-24073 \alpha^{3}+\right.$ $\left.44962 \alpha^{2}-26907 \alpha+4800\right) n+62500 \alpha^{4}+1090000 \alpha^{3}-2334917 \alpha^{2}+1499264 \alpha-303575=\varphi_{29}(n)$. Since 
$\varphi_{29}^{\prime \prime}(n)=2500 \alpha(27-31 \alpha)(1-\alpha)>0$, we have $\varphi_{29}^{\prime}(n) \geq \varphi_{29}^{\prime}(24)=25\left(1050 \alpha^{4}+50327 \alpha^{3}-94238 \alpha^{2}+\right.$ $37893 \alpha+4800)>0$, and thus $15625 Y_{3}(x) \geq \varphi_{29}(n) \geq \varphi_{29}(24)=692500 \alpha^{4}+8966200 \alpha^{3}-17117717 \alpha^{2}+$ $4795064 \alpha+2576425>0$. This completes the proof of $(2.5)$.

Case 6. $0.81<\alpha \leq 0.87$ and $n=23$. Since $(\alpha-1)(n-3)-1<0$ and $625 Y_{2}(20.8)=-2\left(2409750 \alpha^{4}-\right.$ $\left.11557450 \alpha^{3}+19284595 \alpha^{2}-13475955 \alpha+3339258\right)>0$, we have $\beta_{1}>20.8$, and thus it suffices to show that

$$
\text { If } x \geq 20.8 \text {, then } Y_{3}(x)>0 \text {. }
$$

Since $Y_{3}^{\prime \prime \prime}(x)=6 \varphi_{18}(\alpha)>0$, we have $5 Y_{3}^{\prime \prime}(x) \geq 5 Y_{3}^{\prime \prime}(20.8)=-690 \alpha^{3}+1552 \alpha^{2}-474 \alpha+20>0$, and thus $25 Y_{3}^{\prime}(x) \geq 25 Y_{3}^{\prime}(20.8)=1150 \alpha^{4}-34710 \alpha^{3}+46314 \alpha^{2}-13373 \alpha+1205>0$, which implies that $125 Y_{3}(x) \geq$ $125 Y_{3}(20.8)=2\left(2550 \alpha^{4}+34465 \alpha^{3}-63984 \alpha^{2}+16868 \alpha+9705\right)>0$. Thus, (2.6) holds.

Claim 8. If $x \geq n-2.5$, then $\Phi_{3}^{\prime}(x)>0$.

Proof. Since $\Phi_{3}^{\prime \prime \prime}(x)=24 x-6(2 \alpha+1) n+18 \alpha+30 \geq \Phi_{3}^{\prime \prime \prime}(n-2.5)=(18-12 \alpha) n+18 \alpha-30 \geq(18-12 \alpha) 23+18 \alpha-$ $30=6(64-43 \alpha)>0$, we have $\Phi_{3}^{\prime \prime}(x) \geq \Phi_{3}^{\prime \prime}(n-2.5)=2(1-\alpha)(3-\alpha) n^{2}-\left(4 \alpha^{2}-28 \alpha+21\right) n-4 \alpha^{2}-29 \alpha+22=$ $\varphi_{30}(n)$. Note that $\varphi_{30}^{\prime \prime}(n)=4(1-\alpha)(3-\alpha)>0$. Thus, $\varphi_{30}^{\prime}(n) \geq \varphi_{30}^{\prime}(23)=88 \alpha^{2}-340 \alpha+255>0$, and so $\Phi_{3}^{\prime \prime}(x) \geq \varphi_{30}(n) \geq \varphi_{30}(23)=962 \alpha^{2}-3617 \alpha+2713>0$, which implies that $4 \Phi_{3}^{\prime}(x) \geq 4 \Phi_{3}^{\prime}(n-2.5)=$ $4(1-\alpha)^{2} n^{3}+4(1-\alpha)\left(\alpha^{2}+6 \alpha-6\right) n^{2}+\left(16 \alpha^{3}+52 \alpha^{2}-146 \alpha+77\right) n-96 \alpha^{2}+213 \alpha-115=\varphi_{31}(n)$. Since $\varphi_{31}^{\prime \prime}(n)=8(1-\alpha)\left(3(1-\alpha) n+\alpha^{2}+6 \alpha-6\right) \geq \varphi_{31}^{\prime \prime}(23)=8(1-\alpha)\left(\alpha^{2}-63 \alpha+63\right)>0$, we have $\varphi_{31}^{\prime}(n) \geq \varphi_{31}^{\prime}(23)=$ $-168 \alpha^{3}+5480 \alpha^{2}-10634 \alpha+5321>0$, and so $\varphi_{31}(n) \geq \varphi_{31}(23)=-1748 \alpha^{3}+39188 \alpha^{2}-75089 \alpha+37628>$ 0 .

By (2.2) and Claim 7, we have $(1+(1-\alpha)(n-3)) \Phi_{3}\left(\beta_{1}\right)=Y_{3}\left(\beta_{1}\right)>0$, and thus $\Phi_{3}\left(\beta_{1}\right)>0$. Combining this with $\beta_{1}>n-2.26>n-2.5$ by Claim 3, if $x \geq \beta_{1}$, then $\Phi_{3}(x) \geq \Phi_{3}\left(\beta_{1}\right)>0$ by Claim 8 , which implies that $\beta_{1}>\rho_{\alpha}\left(\overline{B_{4}}\right)$.

On the other hand, we have $\Phi_{2}\left(\beta_{1}\right)=\left(\beta_{1}-n+2\right) \Phi_{3}\left(\beta_{1}\right)<0$ (as $\beta_{1}<n-2$ by Claim 4), which implies that $\beta_{1}<\rho_{\alpha}\left(\overline{B_{3}}\right)$. Now, we can conclude that $\rho_{\alpha}\left(\overline{B_{4}}\right)<\beta_{1}<\rho_{\alpha}\left(\overline{B_{3}}\right)$.

Proof of Theorem 1.6. By Lemma 2.1, either $\overline{B_{3}}$ or $\overline{B_{4}}$ is the $\rho_{\alpha}$-maximal graph of $\overline{\mathbb{G}}(n, n-2 ; 2)$. Since $B_{1}$ and $B_{2}$ are all bicyclic graphs with $n$ vertices and maximum degree $n-1$, by Theorems 1.2 (3) and 1.3, to complete the proof of Theorem 1.6, it suffices to show that: If $n \geq 23$ and $0 \leq \alpha \leq 0.87$, then $\rho_{\alpha}\left(\overline{B_{4}}\right)<\rho_{\alpha}\left(\overline{B_{3}}\right)<\rho_{\alpha}\left(\overline{B_{2}}\right)<$ $\rho_{\alpha}\left(\overline{B_{1}}\right)$. Now, the result follows from Lemmas 2.2-2.3.

\section{The proof of Theorem 1.7}

This section is dedicated to the proof of Theorem 1.7.

Lemma 3.1. [3] If $n \geq 7$ and $0 \leq \alpha<1$, then $\rho_{\alpha}\left(\overline{S_{5}}\right)<\rho_{\alpha}\left(\overline{S_{4}}\right)<\rho_{\alpha}\left(\overline{S_{3}}\right)<\min \left\{\rho_{\alpha}\left(\overline{S_{2}}\right), \rho_{\alpha}\left(\overline{S_{1}}\right)\right\}$.

By an elementary computation, $\rho_{\alpha}\left(\overline{S_{7}}\right), \rho_{\alpha}\left(\overline{S_{6}}\right)$, and $\rho_{\alpha}\left(\overline{S_{5}}\right)$ are equal to the maximum root of $\Phi_{6}(x), \Phi_{7}(x)$ and $\Phi_{8}(x)$, respectively, where

$$
\begin{aligned}
& \Phi_{6}(x)=x^{5}-((3 \alpha+1) n-6 \alpha-6) x^{4}+\left(3 \alpha(\alpha+1) n^{2}-\left(11 \alpha^{2}+20 \alpha+4\right) n+4 \alpha^{2}+26 \alpha+17\right) x^{3} \\
& -\left(\alpha^{2}(\alpha+3) n^{3}-\alpha\left(4 \alpha^{2}+21 \alpha+8\right) n^{2}-\left(3 \alpha^{3}-28 \alpha^{2}-55 \alpha-1\right) n+6 \alpha^{3}+36 \alpha^{2}-96 \alpha-2\right) x^{2} \\
& +\left(\alpha^{3} n^{4}+\alpha^{2}\left(\alpha^{2}-6 \alpha-4\right) n^{3}-\alpha^{2}\left(7 \alpha^{2}+8 \alpha-36\right) n^{2}+\left(10 \alpha^{4}+81 \alpha^{3}-77 \alpha^{2}-15 \alpha+5\right) n\right. \\
& \left.-56 \alpha^{3}-33 \alpha^{2}+78 \alpha-25\right) x-\alpha(n-4)(n-5)\left((n-2) \alpha^{2}+\alpha-1\right)((n+1) \alpha-3), \\
& \Phi_{7}(x)=x^{5}-((3 \alpha+1) n-7 \alpha-5) x^{4}+\left(3 \alpha(\alpha+1) n^{2}-\left(13 \alpha^{2}+19 \alpha+3\right) n+5 \alpha^{2}+28 \alpha+12\right) x^{3}
\end{aligned}
$$




$$
\begin{aligned}
& -\left(\alpha^{2}(\alpha+3) n^{3}-\alpha\left(5 \alpha^{2}+22 \alpha+6\right) n^{2}-\left(3 \alpha^{3}-34 \alpha^{2}-48 \alpha+2\right) n+7 \alpha^{3}+39 \alpha^{2}-110 \alpha+14\right) x^{2} \\
& +\left(\alpha^{3} n^{4}+\alpha^{2}\left(\alpha^{2}-7 \alpha-3\right) n^{3}-\alpha\left(8 \alpha^{3}+5 \alpha^{2}-35 \alpha+3\right) n^{2}+\left(12 \alpha^{4}+89 \alpha^{3}-103 \alpha^{2}+5 \alpha+5\right) n\right. \\
& \left.-68 \alpha^{3}-8 \alpha^{2}+68 \alpha-28\right) x-(n-6)\left((n-2) \alpha^{2}+\alpha-1\right)\left(\alpha^{2} n^{2}-\alpha(3 \alpha+2) n-4 \alpha^{2}+12 \alpha-2\right), \\
& \text { and } \Phi_{8}(x)=x^{2}-((\alpha+1) n-\alpha-4) x+(n+1)(n-4) \alpha+10-2 n .
\end{aligned}
$$

In what follows, we shall prove that $\rho_{\alpha}\left(\overline{S_{7}}\right)<\rho_{\alpha}\left(\overline{S_{6}}\right)<\rho_{\alpha}\left(\overline{S_{5}}\right)$.

Lemma 3.2. If $n \geq 35$ and $0 \leq \alpha \leq 0.8$, then $\rho_{\alpha}\left(\overline{S_{6}}\right)<\rho_{\alpha}\left(\overline{S_{5}}\right)$.

Proof. It can be checked that

$$
\Phi_{7}(x)=\Phi_{8}(x) \Psi_{3}(x)+Y_{4}(x),
$$

where $\Psi_{3}(x)=x^{3}-((2 n-6) \alpha-1) x^{2}+\left(\left(n^{2}-5 n-1\right) \alpha^{2}-(n-7) \alpha-2\right) x-(n-1)(n-6) \alpha^{3}+3(n-6) \alpha^{2}-4(n-$ 7) $\alpha+2 n-16$, and $Y_{4}(x)=(1-\alpha) \Psi_{4}(x)$, where $\Psi_{4}(x)=\left((n-6) \alpha^{3}+(n-4)(n-6) \alpha^{2}-\left(3 n^{2}-26 n+50\right) \alpha+(2 n-\right.$ $7)(n-8)) x-(n-6)(n-4)(n+1) \alpha^{3}+(n-6)\left(3 n^{2}-5 n-26\right) \alpha^{2}-\left(2 n^{3}-16 n^{2}-28 n+256\right) \alpha+4 n^{2}-54 n+172$.

Claim 9. $\rho_{\alpha}\left(\overline{S_{5}}\right)>n-2.5$.

Proof. Since $4 \Phi_{8}(n-2.5)=-2(1-\alpha) n-26 \alpha+25 \leq-70(1-\alpha)-26 \alpha+25=44 \alpha-45<0$, we have $\rho_{\alpha}\left(\overline{S_{5}}\right)>n-2.5$, as $\Phi_{8}(+\infty)>0$.

Claim 10. If $x \geq n-2.5$, then $\Psi_{3}(x)>0$.

Proof. When $x \geq n-2.5$, since $\Psi_{3}^{\prime \prime}(x)=6 x-4(n-3) \alpha+2 \geq 6(n-2.5)-4(n-3) \alpha+2=(6-4 \alpha) n+12 \alpha-13>$ $n-13>0,4 \Psi_{3}^{\prime}(x) \geq 4 \Psi_{3}^{\prime}(n-2.5)=\left(4 n^{2}-20 n-4\right) \alpha^{2}-\left(16 n^{2}-84 n+92\right) \alpha+12 n^{2}-52 n+47=\varphi_{1}(\alpha)$. Since $5 \varphi_{1}^{\prime}(\alpha)=20\left(\left(2 n^{2}-10 n-2\right) \alpha-4 n^{2}+21 n-23\right) \leq 5 \varphi_{1}^{\prime}(0.8)=4\left(-12 n^{2}+65 n-123\right)<0$, we have $100 \Psi_{3}^{\prime}(x) \geq 25 \varphi_{1}(\alpha) \geq 25 \varphi_{1}(0.8)=44 n^{2}+60 n-729>0$.

Thus, $8 \Psi_{3}(x) \geq 8 \Psi_{3}(n-2.5)=-8(n-1)(n-6) \alpha^{3}+\left(8 n^{3}-60 n^{2}+116 n-124\right) \alpha^{2}-\left(16 n^{3}-120 n^{2}+296 n-\right.$ $384) \alpha+8 n^{3}-52 n^{2}+110 n-163=\varphi_{2}(\alpha)$. Since $\varphi_{2}^{\prime \prime}(\alpha)=\left(-48 n^{2}+336 n-288\right) \alpha+16 n^{3}-120 n^{2}+232 n-248 \geq$ $0.8\left(-48 n^{2}+336 n-288\right)+16 n^{3}-120 n^{2}+232 n-248=1.6\left(10 n^{3}-99 n^{2}+313 n-299\right)>0$, we have $25 \varphi_{2}^{\prime}(\alpha) \leq 25 \varphi_{2}^{\prime}(0.8)=-8\left(10 n^{3}-27 n^{2}+9 n-292\right)<0$, and thus $1000 \Psi_{3}(x) \geq 125 \varphi_{2}(\alpha) \geq 125 \varphi_{2}(0.8)=$ $40 n^{3}+188 n^{2}-2986 n+5033>0$.

Claim 11. If $x \geq n-2.5$, then $\Psi_{4}(x)>0$.

Proof. When $x \geq n-2.5, \Psi_{4}^{\prime}(x)=(n-6) \alpha^{3}+(n-4)(n-6) \alpha^{2}-\left(3 n^{2}-26 n+50\right) \alpha+(2 n-7)(n-8)=\varphi_{3}(\alpha)$. Since $\varphi_{3}^{\prime \prime}(\alpha)=2(n-6)(3 \alpha+n-4)>0$, we have $25 \varphi_{3}^{\prime}(\alpha) \leq 25 \varphi_{3}^{\prime}(0.8)=-35 n^{2}+298 n-578<0$, and thus $125 \Psi_{4}^{\prime}(x)=125 \varphi_{3}(\alpha) \geq 125 \varphi_{3}(0.8)=30 n^{2}-1011 n+3536>0$.

Thus, $2 \Psi_{4}(x) \geq 2 \Psi_{4}(n-2.5)=-(n-6)\left(2 n^{2}-8 n-3\right) \alpha^{3}+(n-6)\left(8 n^{2}-23 n-32\right) \alpha^{2}-\left(10 n^{3}-99 n^{2}+\right.$ $174 n+262) \alpha+4 n^{3}-48 n^{2}+119 n+64=\varphi_{4}(\alpha)$. Since $5 \varphi_{4}^{\prime \prime}(\alpha)=10(n-6)\left(\left(-6 n^{2}+24 n+9\right) \alpha+8 n^{2}-\right.$ $23 n-32) \geq 10(n-6)\left(0.8\left(-6 n^{2}+24 n+9\right)+8 n^{2}-23 n-32\right)=2(n-6)\left(16 n^{2}-19 n-124\right)>0$, we have $25 \varphi_{4}^{\prime}(\alpha) \leq 25 \varphi_{4}^{\prime}(0.8)=-26 n^{3}+595 n^{2}-2270 n+266<0$, and thus $250 \Psi_{4}(x) \geq 125 \varphi_{4}(\alpha) \geq 125 \varphi_{4}(0.8)=$ $12 n^{3}-500 n^{2}+3075 n-3992>0$.

By Claims 9-11 and (3.1), when $x \geq \rho_{\alpha}\left(\overline{S_{5}}\right)>n-2.5$, we have $\Phi_{7}(x)=\Phi_{8}(x) \Psi_{3}(x)+Y_{4}(x)>0$, and thus $\rho_{\alpha}\left(S_{5}\right)>\rho_{\alpha}\left(S_{6}\right)$.

By $\Phi_{6}(x)$ and $\Phi_{7}(x)$, we can deduce the following Lemma 3.3.

Lemma 3.3. If $n \geq 35$ and $0 \leq \alpha \leq 0.8$, then $\rho_{\alpha}\left(\overline{S_{7}}\right)<\rho_{\alpha}\left(\overline{S_{6}}\right)$. 
Proof. It can be checked that

$$
\Phi_{6}(x)=\Phi_{7}(x)+Y_{5}(x), \text { and } \Phi_{7}(x)=Y_{5}(x) \Psi_{5}(x)+Y_{6}(x)
$$

where $Y_{5}(x)=(1-\alpha) x^{4}+\left(\left(2 \alpha^{2}-\alpha-1\right) n-\alpha^{2}-2 \alpha+5\right) x^{3}+\left(\alpha(\alpha+2)(1-\alpha) n^{2}-(3 \alpha+1)(3-2 \alpha) n+\alpha^{3}+3 \alpha^{2}-\right.$ $14 \alpha+16) x^{2}+\left(\alpha^{2}(\alpha-1) n^{3}+\alpha\left(\alpha^{3}-3 \alpha^{2}+\alpha+3\right) n^{2}-2 \alpha\left(\alpha^{3}+4 \alpha^{2}-13 \alpha+10\right) n-(1-\alpha)\left(12 \alpha^{2}-13 \alpha-3\right)\right) x-$ $\left((n-2) \alpha^{2}+\alpha-1\right)\left(\alpha(\alpha-1) n^{2}-\left(3 \alpha^{2}-3 \alpha-2\right) n-4 \alpha^{2}+12 \alpha-12\right),(1-\alpha)^{2} \Psi_{5}(x)=x(1-\alpha)-\alpha((1-\alpha) n+6 \alpha-4)$ and $(1-\alpha)^{2} Y_{6}(x)=\Psi_{6}(x)$ with $\Psi_{6}(x)=2(2 \alpha-1)(3 \alpha(\alpha-1)+2) x^{3}-\left(2\left(6 \alpha^{4}-4 \alpha^{3}+\alpha^{2}+2 \alpha-1\right) n+\alpha^{5}-\right.$ $\left.\alpha^{4}-48 \alpha^{3}+86 \alpha^{2}-67 \alpha+17\right) x^{2}+\left(2 \alpha\left(5 \alpha^{3}-6 \alpha^{2}+4 \alpha-1\right) n^{2}+\left(11 \alpha^{5}-45 \alpha^{4}+54 \alpha^{3}-30 \alpha^{2}-\alpha+3\right) n+2(1-\right.$ $\left.\alpha)\left(2 \alpha^{4}+19 \alpha^{3}-35 \alpha^{2}+30 \alpha-8\right)\right) x-2\left(\left(5 \alpha^{3}-6 \alpha^{2}+4 \alpha-1\right) n-16 \alpha^{3}+30 \alpha^{2}-24 \alpha+6\right)\left(\alpha+\alpha^{2}(n-2)-1\right)$.

Claim 12. If $x \geq n-2$, then $Y_{5}(x)>0$.

Proof. When $x \geq n-2$, since $Y_{5}^{(4)}(x)=24(1-\alpha)>0$, we have $Y_{5}^{\prime \prime \prime}(x) \geq Y_{5}^{\prime \prime \prime}(n-2)=6\left(\left(2 \alpha^{2}-5 \alpha+3\right) n-\alpha^{2}+6 \alpha-3\right) \geq$ $6\left(35\left(2 \alpha^{2}-5 \alpha+3\right)-\alpha^{2}+6 \alpha-3\right)=6\left(69 \alpha^{2}-169 \alpha+102\right)>0$.

Thus, $Y_{5}^{\prime \prime}(x) \geq Y_{5}^{\prime \prime}(n-2)=2\left((1-\alpha)^{2}(3-\alpha) n^{2}-\left(9 \alpha^{2}-17 \alpha+6\right) n+\alpha^{3}+9 \alpha^{2}-26 \alpha+10\right)=2 \varphi_{1}(n)$. Since $\varphi_{1}^{\prime \prime}(n)=2(1-\alpha)^{2}(3-\alpha)>0$, we have $\varphi_{1}^{\prime}(n) \geq \varphi_{1}^{\prime}(35)=-70 \alpha^{3}+341 \alpha^{2}-473 \alpha+204>0$, and thus $0.5 Y_{5}^{\prime \prime}(x) \geq \varphi_{1}(n) \geq \varphi_{1}(35)=-1224 \alpha^{3}+5819 \alpha^{2}-8006 \alpha+3475>0$.

This implies that $Y_{5}^{\prime}(x) \geq Y_{5}^{\prime}(n-2)=(1-\alpha) \varphi_{2}(n)$, where $\varphi_{2}(n)=(1-\alpha)^{2} n^{3}-\left(\alpha^{3}+2 \alpha^{2}-8 \alpha+3\right) n^{2}+$ $\left(2 \alpha^{3}+8 \alpha^{2}-36 \alpha+20\right) n-(1-\alpha)(33-8 \alpha)$. Since $\varphi_{2}^{\prime \prime \prime}(n)=6(1-\alpha)^{2}>0$, we have $\varphi_{2}^{\prime \prime}(n) \geq \varphi_{2}^{\prime \prime}(35)=$ $-2 \alpha^{3}+206 \alpha^{2}-404 \alpha+204>0$, and thus $\varphi_{2}^{\prime}(n) \geq \varphi_{2}^{\prime}(35)=-68 \alpha^{3}+3543 \alpha^{2}-6826 \alpha+3485>0$. Now, we can conclude that $\varphi_{2}(n) \geq \varphi_{2}(35)=-1155 \alpha^{3}+40697 \alpha^{2}-77169 \alpha+39867>0$, which implies that $Y_{5}^{\prime}(x)>0$.

Thus, $Y_{5}(x) \geq Y_{5}(n-2)=(1-\alpha)^{2} \varphi_{3}(n)$, where $\varphi_{3}(n)=\left(\alpha^{2}-9 \alpha+10\right) n^{2}+\left(2 \alpha^{2}+25 \alpha-35\right) n-8 \alpha^{2}-8 \alpha+22$. Since $\varphi_{3}^{\prime \prime}(n)=2\left(\alpha^{2}-9 \alpha+10\right)>0, \varphi_{3}^{\prime}(n) \geq \varphi_{3}^{\prime}(35)=72 \alpha^{2}-605 \alpha+665>0$, and so $\varphi_{3}(n) \geq \varphi_{3}(35)=$ $1287 \alpha^{2}-10158 \alpha+11047>0$.

Claim 13. $Y_{5}(n-2.5)<0$.

Proof. It can be checked that $16 Y_{5}(n-2.5)=\varphi_{4}(n)=8(\alpha-1)^{3} n^{3}-4(1-\alpha)\left(2 \alpha^{3}-45 \alpha^{2}+96 \alpha-49\right) n^{2}+\left(48 \alpha^{4}+\right.$ $\left.384 \alpha^{3}-1720 \alpha^{2}+2046 \alpha-750\right) n-128 \alpha^{4}+68 \alpha^{3}+910 \alpha^{2}-1541 \alpha+663$. Since $\varphi_{4}^{\prime \prime \prime}(n)=48(\alpha-1)^{3}<0$, we have $\varphi_{4}^{\prime \prime}(n) \leq \varphi_{4}^{\prime \prime}(35)=8(\alpha-1)\left(2 \alpha^{3}+165 \alpha^{2}-324 \alpha+161\right)<0$. Thus, $\varphi_{4}^{\prime}(n) \leq \varphi_{4}^{\prime}(35)=608 \alpha^{4}+$ $16624 \alpha^{3}-50440 \alpha^{2}+49646 \alpha-16430<0$. Now, we can conclude that $16 Y_{5}(n-2.5)=\varphi_{4}(n) \leq \varphi_{4}(35)=$ $11352 \alpha^{4}+126208 \alpha^{3}-397390 \alpha^{2}+388569 \alpha-128487<0$.

Let $\beta_{1}$ be the maximum roots of $Y_{5}(x)$. By Claims 12 and 13, we have

$$
n-3<n-2.5<\beta_{1}<n-2 .
$$

For the case $35 \leq n \leq 49$, we can slightly improves (3.3) to

Claim 14. If $35 \leq n \leq 49$, then $n-2.5<\beta_{1}<n-2.15$.

Proof. From Claim 13, it suffices to show that

$$
\text { If } x \geq n-2.15 \text {, then } Y_{5}(x)>0 \text {. }
$$

When $x \geq n-2.15$, since $Y_{5}^{(4)}(x)=24(1-\alpha)>0$, we have $5 Y_{5}^{\prime \prime \prime}(x) \geq 5 Y_{5}^{\prime \prime \prime}(n-2.15)=30(1-\alpha)(3-2 \alpha) n-$ $30 \alpha^{2}+198 \alpha-108 \geq 1050(1-\alpha)(3-2 \alpha)-30 \alpha^{2}+198 \alpha-108=6\left(345 \alpha^{2}-842 \alpha+507\right)>0$.

Thus, $100 Y_{5}^{\prime \prime}(x) \geq 100 Y_{5}^{\prime \prime}(n-2.15)=200(3-\alpha)(1-\alpha)^{2} n^{2}-10\left(198 \alpha^{2}-385 \alpha+147\right) n+200 \alpha^{3}+1890 \alpha^{2}-$ $5767 \alpha+2297=\varphi_{5}(n)$. Since $\varphi_{5}^{\prime \prime}(n)=400(3-\alpha)(1-\alpha)^{2}>0$, we have $\varphi_{5}^{\prime}(n) \geq \varphi_{5}^{\prime}(35)=-14000 \alpha^{3}+$ 
$68020 \alpha^{2}-94150 \alpha+40530>0$, and thus $100 Y_{5}^{\prime \prime}(x) \geq \varphi_{5}(n) \geq \varphi_{5}(35)=-244800 \alpha^{3}+1157590 \alpha^{2}-$ $1586017 \alpha+685847>0$.

This implies that $2000 Y_{5}^{\prime}(x) \geq 2000 Y_{5}^{\prime}(n-2.15)=\varphi_{6}(n)$, where $\varphi_{6}(n)=2000(1-\alpha)^{3} n^{3}-200(1-\alpha)(2 \alpha-$ 1) $\left(5 \alpha^{2}+14 \alpha-39\right) n^{2}-5\left(800 \alpha^{4}+2400 \alpha^{3}-18734 \alpha^{2}+24575 \alpha-8801\right) n+15400 \alpha^{3}-103535 \alpha^{2}+164437 \alpha-$ 72432. Note that $\varphi_{6}^{\prime \prime \prime}(n)=12000(1-\alpha)^{3}>0$. Thus, $\varphi_{6}^{\prime \prime}(n) \geq \varphi_{6}^{\prime \prime}(35)=400(1-\alpha)\left(-10 \alpha^{3}+1027 \alpha^{2}-2008 \alpha+\right.$ 1011) $>0$, which implies that $\varphi_{6}^{\prime}(n) \geq \varphi_{6}^{\prime}(35)=136000 \alpha^{4}-7180000 \alpha^{3}+20533670 \alpha^{2}-20338875 \alpha+$ $6848005=5\left(27200 \alpha^{4}-1436000 \alpha^{3}+4106734 \alpha^{2}-4067775 \alpha+1369601\right)>0$. Thus, $2000 Y_{5}^{\prime}(x) \geq \varphi_{6}(n) \geq$ $\varphi_{6}(35)=2310000 \alpha^{4}-82969600 \alpha^{3}+232249915 \alpha^{2}-229291188 \alpha+77662743>0$.

Now, we can conclude that $160000 Y_{5}(x) \geq 160000 Y_{5}(n-2.15)=\varphi_{7}(\alpha)$, where $\varphi_{7}(\alpha)=8000(17 n+$ $80)(n-2) \alpha^{4}+400\left(60 n^{3}-4469 n^{2}+8760 n+2729\right) \alpha^{3}-20\left(3600 n^{3}-244900 n^{2}+718474 n-300447\right) \alpha^{2}+$ $\left(72000 n^{3}-4929200 n^{2}+16607900 n-10192921\right) \alpha-24000 n^{3}+1682800 n^{2}-6103220 n+4349701$. Since $\varphi_{7}^{(4)}(\alpha)=192000(17 n+80)(n-2)>0$ and $35 \leq n \leq 49, \varphi_{7}^{\prime \prime \prime}(\alpha) \leq \varphi_{7}^{\prime \prime \prime}(0.8)=2400\left(60 n^{3}-3381 n^{2}+\right.$ $11704 n-7511)<0$, and so $\varphi_{7}^{\prime \prime}(\alpha) \geq \varphi_{7}^{\prime \prime}(0.8)=-40\left(720 n^{3}-56500 n^{2}+227338 n-185679\right)>0$, and so $\varphi_{7}^{\prime}(\alpha) \leq \varphi_{7}^{\prime}(0.8)=2880 n^{3}-246064 n^{2}+1098076 n-1104185<0$. This implies that $800000 Y_{5}(x) \geq 5 \varphi_{7}(\alpha) \geq$ $5 \varphi_{7}(0.8)=-960 n^{3}+73072 n^{2}-342932 n+378485>0$. Thus (3.4) holds.

Claim 15. $\Phi_{7}\left(\beta_{1}\right)=Y_{6}\left(\beta_{1}\right)<0$.

Proof. Let $\varphi_{8}(\alpha)=(2 \alpha-1)(3 \alpha(\alpha-1)+2)$. Since $(1-\alpha)^{2} Y_{6}(x)=\Psi_{6}(x)$ and $\beta_{1}>n-3$ by (3.3), to complete the proof, it suffices to show that

$$
\text { If } x \geq n-3 \text {, then } \Psi_{6}(x)<0 .
$$

Case 1. $0 \leq \alpha<0$.5. Let $\varphi_{9}(\alpha)=-12 \alpha^{4}+44 \alpha^{3}-56 \alpha^{2}+38 \alpha-10$. Since $\varphi_{9}^{\prime \prime}(\alpha)=-8(2-3 \alpha)(7-6 \alpha)<0$, we have $\varphi_{9}^{\prime}(\alpha)=2\left(-24 \alpha^{3}+66 \alpha^{2}-56 \alpha+19\right)>\varphi_{9}^{\prime}(0.5)=9>0$, and thus $\varphi_{9}(\alpha)<\varphi_{9}(0.5)=-0.25<0$. When $x \geq n-3$, since $\Psi_{6}^{\prime \prime \prime}(x)=12 \varphi_{8}(\alpha)<0$, we have $0.5 \Psi_{6}^{\prime \prime}(x) \leq 0.5 \Psi_{6}^{\prime \prime}(n-3)=\varphi_{9}(\alpha) n-\alpha^{5}+\alpha^{4}-60 \alpha^{3}+76 \alpha^{2}-59 \alpha+19 \leq$ $35 \varphi_{9}(\alpha)-\alpha^{5}+\alpha^{4}-60 \alpha^{3}+76 \alpha^{2}-59 \alpha+19=-\alpha^{5}-419 \alpha^{4}+1480 \alpha^{3}-1884 \alpha^{2}+1271 \alpha-331<0$.

Now, we have $\Psi_{6}^{\prime}(x) \leq \Psi_{6}^{\prime}(n-3)=(9 n+2) \alpha^{5}-\left(14 n^{2}-29 n+40\right) \alpha^{4}+\left(40 n^{2}-114 n+144\right) \alpha^{3}-\left(50 n^{2}-\right.$ $134 n+100) \alpha^{2}+\left(32 n^{2}-95 n+52\right) \alpha-8 n^{2}+29 n-22=\varphi_{10}(\alpha)$. Since $\varphi_{10}^{(5)}(\alpha)=120(9 n+2)>0$ and $n \geq 35$, we have $\varphi_{10}^{(4)}(\alpha)<\varphi_{10}^{(4)}(0.5)=-12\left(28 n^{2}-103 n+70\right)<0$, and thus $\varphi_{10}^{\prime \prime \prime}(\alpha)>\varphi_{10}^{\prime \prime \prime}(0.5)=3\left(24 n^{2}-67 n+138\right)>0$. This implies that $2 \varphi_{10}^{\prime \prime}(\alpha)<2 \varphi_{10}^{\prime \prime}(0.5)=-(11 n+18)(4 n-13)<0$ and so $16 \varphi_{10}^{\prime}(\alpha)>16 \varphi_{10}^{\prime}(0.5)=80 n^{2}-$ $467 n+650>0$. Thus, $32 \Psi_{6}^{\prime}(x) \leq 32 \varphi_{10}(\alpha)<32 \varphi_{10}(0.5)=-12 n^{2}+91 n-174<0$.

Now, we can conclude that $\Psi_{6}(x) \leq \Psi_{6}(n-3)=(21 n-61) \alpha^{5}-\left(2 n^{3}-10 n^{2}+107 n-263\right) \alpha^{4}+\left(8 n^{3}-26 n^{2}+\right.$ $140 n-404) \alpha^{3}-\left(12 n^{3}-36 n^{2}+64 n-234\right) \alpha^{2}+\left(8 n^{3}-30 n^{2}+29 n-63\right) \alpha-2 n^{3}+10 n^{2}-15 n+15=\varphi_{11}(\alpha)$. Since $\varphi_{11}^{(5)}(\alpha)=120(21 n-61)>0$, we have $\varphi_{11}^{(4)}(\alpha) \leq \varphi_{11}^{(4)}(0.5)=-12\left(4 n^{3}-20 n^{2}+109 n-221\right)<0$, and so $\varphi_{11}^{\prime \prime \prime}(\alpha)>\varphi_{11}^{\prime \prime \prime}(0.5)=3\left(8 n^{3}-12 n^{2}-43 n-61\right)>0$, which implies that $2 \varphi_{11}^{\prime \prime}(\alpha)<2 \varphi_{11}^{\prime \prime}(0.5)=$ $-12 n^{3}+48 n^{2}+47 n-215<0$. From this, it follows that $16 \varphi_{11}^{\prime}(\alpha)>16 \varphi_{11}^{\prime}(0.5)=16 n^{3}-136 n^{2}+369 n-313>0$ and thus $32 \Psi_{6}(x) \leq 32 \varphi_{11}(\alpha)<32 \varphi_{11}(0.5)=-4 n^{3}+44 n^{2}-161 n+193<0$. In this case, (3.5) holds.

Case 2. $\alpha=0.5$. Then, $32 \Psi_{6}(x)=(-8 n+33) x^{2}+\left(4 n^{2}-23 n+24\right) x-2(n-4)^{2}=\varphi_{12}(x)$. When $x \geq n-3$, since $32 \Psi_{6}^{\prime}(x)=(66-16 n) x+4 n^{2}-23 n+24 \leq(66-16 n)(n-3)+4 n^{2}-23 n+24=-12 n^{2}+91 n-174<0$, we have $32 \Psi_{6}(x)=\varphi_{12}(x) \leq \varphi_{12}(n-3)=-4 n^{3}+44 n^{2}-161 n+193<0$, and thus (3.5) holds.

Case 3. $0.5<\alpha \leq 0.8$. To prove Claim (3.5), by Claim 14 and (3.3), since $\Psi_{6}(-\infty)<0$ and $\Psi_{6}(+\infty)>0$, it suffices to show that $\Psi_{6}(\alpha)>0, \max \left\{\Psi_{6}(n-2.5), \Psi_{6}(n-2)\right\}<0$ for $n \geq 50$ and $\max \left\{\Psi_{6}(n-2.5), \Psi_{6}(n-2.15)\right\}<0$ for $35 \leq n \leq 49$.

Since $\varphi_{13}(\alpha)=-\alpha^{4}+13 \alpha^{3}-15 \alpha^{2}+9 \alpha-2>0, \Psi_{6}(\alpha)=(1-\alpha)^{2}\left(\varphi_{13}(\alpha) n-\alpha^{5}+7 \alpha^{4}-53 \alpha^{3}+75 \alpha^{2}-\right.$ $52 \alpha+12) \geq(1-\alpha)^{2}\left(35 \varphi_{13}(\alpha)-\alpha^{5}+7 \alpha^{4}-53 \alpha^{3}+75 \alpha^{2}-52 \alpha+12\right)=(1-\alpha)^{2} \varphi_{14}(\alpha)>0$, as $\varphi_{14}(\alpha)=$ $-\alpha^{5}-28 \alpha^{4}+402 \alpha^{3}-450 \alpha^{2}+263 \alpha-58>0$.

It can be checked that $4 \Psi_{6}(n-2.5)=(102 n-241) \alpha^{5}-\left(8 n^{3}-12 n^{2}+382 n-973\right) \alpha^{4}+\left(32 n^{3}-24 n^{2}+\right.$ $376 n-1382) \alpha^{3}-\left(48 n^{3}-44 n^{2}+44 n-803\right) \alpha^{2}+\left(32 n^{3}-56 n^{2}-36 n-200\right) \alpha-8 n^{3}+24 n^{2}-12 n+33=\varphi_{15}(\alpha)$. Since $\varphi_{15}^{(5)}(\alpha)=120(102 n-241)>0$, we have $\varphi_{15}^{(4)}(\alpha) \leq \varphi_{15}^{(4)}(0.8)=-24(2 n+1)\left(4 n^{2}-8 n-9\right)<0$, and thus $5 \varphi_{15}^{\prime \prime \prime}(\alpha) \geq 5 \varphi_{15}^{\prime \prime \prime}(0.8)=12\left(16 n^{3}+36 n^{2}-484 n+473\right)>0$. This implies that $25 \varphi_{15}^{\prime \prime}(\alpha) \leq 25 \varphi_{15}^{\prime \prime}(0.8)=$ 
$-2\left(48 n^{3}-812 n^{2}+2156 n+285\right)<0$, and so $125 \varphi_{15}^{\prime}(\alpha) \geq 125 \varphi_{15}^{\prime}(0.8)=4\left(8 n^{3}-222 n^{2}+1315 n-2172\right)>0$. Now, we have $12500 \Psi_{6}(n-2.5)=3125 \varphi_{15}(\alpha) \leq 3125 \varphi_{15}(0.8)=-40 n^{3}-40 n^{2}+1588 n-3419<0$.

If $n \geq 50$, then $\Psi_{6}(n-2)=2(1-\alpha)^{2} \varphi_{16}(\alpha)$, where $\varphi_{16}(\alpha)=15(n-2) \alpha^{3}-\left(n^{3}+2 n^{2}+15 n-52\right) \alpha^{2}+$ $\left(2 n^{3}+3 n^{2}-10 n-20\right) \alpha-n^{3}+n^{2}+2 n+4$. Since $\varphi_{16}^{\prime \prime \prime}(\alpha)=90(n-2)>0$, we have $\varphi_{16}^{\prime \prime}(\alpha) \leq \varphi_{16}^{\prime \prime}(0.8)=$ $-2\left(n^{3}+2 n^{2}-21 n+20\right)<0$, and thus $5 \varphi_{16}^{\prime}(\alpha) \geq 5 \varphi_{16}^{\prime}(0.8)=2 n^{3}-n^{2}-26 n+28>0$. Now, we have $25 \varphi_{16}(\alpha) \leq 25 \varphi_{16}(0.8)=-n^{3}+53 n^{2}-198 n+148<0$, and thus $\Psi_{6}(n-2)=2(1-\alpha)^{2} \varphi_{16}(\alpha)<0$.

If $35 \leq n \leq 49$, then $4000 \Psi_{6}(n-2.15)=\varphi_{17}(n)$, where $\varphi_{17}(n)=-8000(1-\alpha)^{4} n^{3}+400(1-\alpha)\left(19 \alpha^{3}-\right.$ $\left.61 \alpha^{2}+4 \alpha+32\right) n^{2}+20\left(5730 \alpha^{5}-18204 \alpha^{4}+14978 \alpha^{3}+1888 \alpha^{2}-4859 \alpha+485\right) n-240090 \alpha^{5}+918890 \alpha^{4}-$ $1270322 \alpha^{3}+771423 \alpha^{2}-211319 \alpha+30284$. Since $\varphi_{17}^{\prime \prime \prime}(n)=-48000(1-\alpha)^{4}<0$, we have $\varphi_{17}^{\prime \prime}(n) \leq \varphi_{17}^{\prime \prime}(35)=$ $800(1-\alpha)\left(2119 \alpha^{3}-6361 \alpha^{2}+6304 \alpha-2068\right)<0$, and so $\varphi_{17}^{\prime}(n) \leq \varphi_{17}^{\prime}(35)=20\left(5730 \alpha^{5}-1514804 \alpha^{4}+\right.$ $\left.6006978 \alpha^{3}-8909112 \alpha^{2}+5835941 \alpha-1424715\right)<0$. This follows that $4000 \Psi_{6}(n-2.15)=\varphi_{17}(n) \leq$ $\varphi_{17}(35)=3770910 \alpha^{5}-364133910 \alpha^{4}+1420414278 \alpha^{3}-2087756977 \alpha^{2}+1354667381 \alpha-326950216<0$. This completes the proof of Claim 15.

From Claim 15, we have $\rho_{\alpha}\left(\overline{S_{6}}\right)>\beta_{1}$. By (3.2), when $x \geq \rho_{\alpha}\left(\overline{S_{6}}\right)$, we can conclude that $\Phi_{6}(x)>0$ and thus $\rho_{\alpha}\left(\overline{S_{7}}\right)<\rho_{\alpha}\left(\overline{S_{6}}\right)$.

The proof of Theorem 1.7: Let $\overline{G_{0}}$ be a $\rho_{\alpha}$-maximal graph of $\overline{\mathbb{G}}(n, n-2 ; 3)$. Then, $G_{0} \in\left\{S_{6}, S_{7}\right\}$ by Lemma 2.1. Note that $\left\{S_{1}, S_{2}, \ldots, S_{5}\right\}$ are all tricyclic graphs with maximum degree $n-1$ and $n$ vertices [4]. Thus, the result follows from Lemmas 3.1-3.3 and Theorem 1.3.

Acknowledgment. The authors would like to thank the referees for their valuable comments and corrections which improved the original manuscript.

Data Availability Statement: Data sharing is not applicable to this article as no datasets were generated or analysed during the current study.

\section{References}

[1] W.H. Haemers, M.R. Oboudi, Universal spectra of the disjoint union of regular graphs, Linear Algebra Appl., 606 (2020), 244-248.

[2] J. Liu, Z. Zhang, Spectral radius of the complement of unicyclic graphs, J. East China Norm. Univ. Natur. Sci. Ed., 5 (2010), 14-19.

[3] M. Liu, C. Chen, S.-G. Guo, J. Peng, T. Chen, The $A_{\alpha}$-spectral radius of dense graphs, paper submitted.

[4] M. Liu, B. Liu, On the spectral radii and the signless Laplacian spectral radii of $c$-cyclic graphs with fixed maximum degree, Linear Algebra Appl., 435 (2011), 3045-3055.

[5] M. Liu, B. Liu, Extremal Theory of Graph Spectrum, University of Kragujevac and Faculty of Science Kragujevac, Kragujevac, 2018.

[6] Z. Stanić, On nested split graphs whose second largest eigenvalue is less than 1, Linear Algebra Appl., 430 (2009), 22002211.

[7] W.-L. Yi, S.-G. Guo, Spectral radius of the complement of bicyclic graphs with $n$ vertices, J. Yancheng Teach. Univ. (Human. \& Soc. Sci. Ed.), 26 (2008), 17-21.

[8] W.-L. Yi, S.-G. Guo, Spectral radius of the complement of tricyclic graphs with $n$ vertices, Appl. Math. J. Chinese Univ. Ser. A, 26 (2011), 223-229.

[9] R. Zhang, S.-G. Guo, The $A_{\alpha}$-spectral radius of the complement of unicyclic and bicyclic graphs with $n$ vertices, Appl. Math. J. Chinese Univ. Ser. A, 36 (2021), 247-252. 